\title{
Il panegirico franco-spagnolo di Loubayssin de la Marque (1639): quale lingua al servizio del potere
}

\section{Simona Munari}

\section{(2) OpenEdition}

1 Journals

\section{Edizione digitale}

URL: https://journals.openedition.org/studifrancesi/22079

DOI: 10.4000/studifrancesi.22079

ISSN: 2421-5856

\section{Editore}

Rosenberg \& Sellier

\section{Edizione cartacea}

Data di pubblicazione: 1 avril 2020

Paginazione: $40-52$

ISSN: 0039-2944

\section{Notizia bibliografica digitale}

Simona Munari, «ll panegirico franco-spagnolo di Loubayssin de la Marque (1639): quale lingua al servizio del potere», Studi Francesi [Online], 190 (LXIV | I) | 2020, online dal 01 avril 2021, consultato il 03 août 2021. URL: http://journals.openedition.org/studifrancesi/22079 ; DOI: https://doi.org/10.4000/ studifrancesi.22079

\section{(c) (i) $\odot$}

Studi Francesi è distribuita con Licenza Creative Commons Attribuzione - Non commerciale - Non opere derivate 4.0 Internazionale. 


\section{Il panegirico franco-spagnolo di Loubayssin de la Marque (1639): quale lingua al servizio del potere}

\section{Abstract}

In 1639, the Parisian bookseller Morlot published a panegyric to Cardinal of Richelieu written by Loubayssin de la Marque, a Gascon gentleman serving the House of Guisa. The text was printed simultaneously in French and Spanish, and it was the final act of an author who put his bilingualism at the service of the French monarchy. Loubayssin's marginal personality, grappling with the fleeting balances of political affairs and with the ambiguous dynamics of courtisanship within the language Babel of the court (where Latin, regional tongues and the languages brought in by foreign queens all coexisted), displays an extremely modern trait in his attempts to find his own role and linguistic belonging, which constantly forces him to question his identity. With Deffy de la langue françoise et de l'espagnole he lays out a cautious strategy to redefine the terms of patronage. Here he resorts to the practice of rhetoric and adulation to reformulate the "récit du roi" through the means of translation, a malleable and flexible tool on the fine line between originality and creative imitation.

Nel 1639, presso il libraio parigino Morlot, François Loubayssin de La Marque pubblica un panegirico al Cardinale di Richelieu in due versioni, francese e spagnola. Scritti uno dopo l'altro in un'epoca in cui l'idea stessa di traduzione è sinonimo di ambiguità e l'atto creativo è concepito come un percorso di imitazione, i due testi abitano uno spazio incerto fra traduzione, imitazione e riscrittura, e situano il loro autore nel novero di quei «bâtisseurs de langues nationales», «artisans de littératures nationales» ${ }^{1}$ stretti nelle mutevoli relazioni di forza che animavano la scena del potere. Nell'epistola al lettore che introduce il Deffy de la langue françoise et de l'espagnole Loubayssin annuncia una traduzione che avrà per titolo Repto entre la lengua española y la francesa:

Passionné de tout entreprendre, pour essayer de parvenir à la gloire de bien meriter: j’ay donc commencé de mettre au jour en la Langue de nos Ennemis, les plus belles actions, et les plus rares Exemples, que la fureur de Mars, et la sagesse de Minerve ayent jamais fait voir en leur plus superbe appareil².

Di Loubayssin, nato verso il 1588 e guascone di origine, si conoscono solo gli elementi biografici riportati nei paratesti della sua opera. E certo che avesse vissuto a lungo a Madrid, anzi conosceva così bene il castigliano, si legge nella prefazione al suo primo romanzo, da sceglierlo come lingua di scrittura. Engaños deste siglo y bistoria sucedida en nuestros tiempos è pubblicato nel 1615 per celebrare il successo diplomatico del doppio matrimonio reale che impone a corte lo spagnolo di Anna

(1) J. Delisle, J. Woodsworth, Les traducteurs dans l'histoire, Ottawa, Presses de l'Université d'Ottawa, 1995 , p. 39 e p. 77

(2) Loubayssin, Deffy de la langue françoise et de l'espagnole, Paris, Morlot,1639, Advertissement. 
d'Austria, sposa di Luigi XIII. Nella dedica a «Enrique» (Henri) de Gondy, duca di Retz, l'autore si augura infatti che il volume sia ben accolto non tanto per il suo valore «que bien se que en si no tiene ningun merito», quanto per la stagione in cui viene presentato:

Al tiempo digo que los Franceses, y los Españoles, dos naciones las mas valerosas de toda la tierra, contractan por medio de un Santo Himeneo, la paz de la Christiandad y la ruyna de los enemigos de la Iglesia. Bien se que no faltarán Aretinos, y Zoylos; porque estamos en un siglo tan depravado y perverso, que todo el mundo quiere corregir los vicios agenos, y nadie quiere enmendar los suyos propios ${ }^{3}$.

$\mathrm{Al}$ «discreto lector» spiega invece di aver voluto dare al libro, in uno scrupolo di verosimiglianza, «no solamente el cuerpo y alma Española: sino tambien el vestido, al modo y trage de su tierra»:

De la orden, y modo de hablar, no te espantes si no corresponde a nuestra lengua: y si huyendo la opinión de muchos, sigo el idioma Español, haziendo hablar a los personages contenidos en mi historia, la verdadera lengua de Castilla, dandoles, la retorica y frasis que aquellos que nacen ribera del dorado Tajo, o del umilde Mançanares, dan, en los discursos que hazen, requebrando a una dama, en el prado de Madrid, o Cigarral de Toledo ${ }^{4}$.

Un'anima e un abito straniero che sono chiaro riferimento alla pratica francese di «habiller à la française» $\mathrm{i}$ testi spagnoli per giustificare rimaneggiamenti che potevano interessare piani diversi, dal cambio di genere a variazioni consistenti di struttura e personaggi.

Le «belles infidèles», secondo la felice definizione di Roger Zuber ${ }^{5}$, erano libere traduzioni di autori per i quali l'originalità si esprimeva in un rapporto di appropriazione ed emulazione di un modello, e celavano spesso contraddizioni e rivalità interne $^{6} . \mathrm{Ma}$ in caso di conflitto internazionale le opere straniere erano trattate alla stregua di «proies de guerre» - puntualizza Cioranescu - per evitare accuse di intesa con il nemico: «Il ne s'agit pas de collaboration, mais d'annexion et d'une façon adroite d'obliger l'ennemi à contribuer au progrès de la France» ${ }^{7}$. Ai traduttori era richiesto di elaborare un'apologetica della lingua francese che trovava spazio in sede paratestuale insieme ad altri elementi, dalle dediche alle note di traduzione, utili a definire la posizione del traduttore nel microcosmo intellettuale in cui svolgeva la sua attività: un'apologetica non certo nuova e non sempre originale, ricorda Siouffi, la cui funzione passa dall'elaborazione di un'idea moderna di lingua vernacolare libera dalla tutela del latino a uno sforzo autoreferenziale di affermazione letteraria sulle altre potenze europee:

La part d'enrôlement politique y est souvent très grande et, comme dans les discours académiques, une sorte de stéréotypie ne tarde pas à se mettre en place, avec ses exagérations convenues, ses formules obligées, ses codes, qui s'appuient sur des «pré-discours» dont il est

(3) Loubayssin, Engaños deste siglo, Paris, Orry, 1615, dedica.

(4) Loubayssin, Engaños cit., Al discreto lector.

(5) R. Zuber, Les «Belles infidèles» et la formation du goût classique, Paris, Albin Michel, 1995 (I ed. 1968).

(6) M. Pavesio, Una ricezione controversa: la comedia spagnola nella Francia del XVII secolo, in S. Torresi

(dir.), Francia e Spagna a confronto, Macerata, eum, 2010, pp. 137-156.

(7) A. Cioranescu, Le masque et le visage. Du baroque espagnol au classicisme français, Genève, Droz, 1983, p. 179. 
difficile d'évaluer aujourd'hui la réception, et qui ne sont en tout cas pas toujours à prendre au premier degré 8 .

Nel corso del Seicento la moda del castigliano aveva definito l'esigenza di nuovi strumenti pedagogici e determinato la fortuna editoriale di grammatiche e dizionari bilingui; inoltre si traducevano e ristampavano opere spagnole a fini didattici'. Il primo romanzo di Loubayssin, pubblicato a Parigi dal libraio Orry, sembra rivolgersi a un pubblico francese, come lascia intuire un passo del prologo:

Si en algo he errado perdoname (amantissimo Lector) que ya sabes, que no solamente yo, sino todos los hombres, somos sujetos a hazer faltas. Serán muchas las que hallarás, y sin esos pobres conceptos, umildes frasis, y muy llanos discursos: Yo lo confiesso, pero por muchas y grandes que sean, no serán bastantes (a lo que creo) de hazerte cerrar la puerta a la misericordia. Hablo con el discreto y pio lector, que del arrogante y presumptuoso, me tengo tan cierto, y siguro, por mas flechas de murmuracion que tire sobre el, teniendote a ti de mi parte, que si tuviera en mi cabeça infusas, todas las ciencias del mundo. Mas porque me dezvanesco tanto en buscar umildades, si escrivo en una lengua estrangera, y sé que el mas ladino Frances que se halle en la lengua Castellana, desde Metz, a Bayona, tendra harto en que entender, a hazer otro tanto como yo he hecho y hare de aqui adelante, si esta vez me acojes con la cortesia que de ti espero ${ }^{10}$.

Sul piano linguistico Loubayssin mostra di avere un buon controllo lessicale e sintattico e non pecca di interferenze se non per qualche gallicismo sul piano grammaticale ${ }^{11}$, mentre la struttura narrativa risulta composita e di difficile classificazione: forzature dell'intreccio, collegamenti imprevedibili, una varietà di stili in cui si alternano componenti picaresche e pastorali. Eppure le disordinate avventure di Don Juan e doña Maria, senza contare l'eco petrarchesca legata alla presenza di «don Francisco y la hermosa Laura», potrebbero rientrare nel quadro dei primi «embrioni pretirsiani $\gg^{12}$ del Don Giovanni, come indica l'Argumento della quinta parte:

La fregona viene puntualmente, a la ora del concierto. Don Juan queriendo ir al lugar que se le avia señalado, encuentra en su camino una vision que le detiene, y le dize que se ha de morir por la mañana. Su conversion, su muerte y otras cosas estrañas, y admirables que sucedieron: por medio de las cuales la Señora de las Estrellas viene a contar al corregidor la historia de sus amores ${ }^{13}$.

Due anni dopo, nel 1617, firmandosi Francisco Loubayssin de la Marca dà alle stampe per i tipi di Guillemot la Historia tragicómica de Henrique de Castro, una vicenda «en cuyos estraños sucesos se veen los varios y prodigiosos efectos de amor y de la guerra $»^{14}$. In una breve nota, Pablo Neruda mette il romanzo in relazione al poema

(8) G. Siouffi, L'apologétique de la langue française et la problématique du «rayonnement»à la fin du XVII siècle, "Littératures Classiques" 76, 2011/3, p. 179.

(9) Cf. S. Collet-Sedola, L'étude de l'espagnol en France à l'époque d'Anne d'Autriche, in C. Mazouer (dir.), L'âge d'or de l'influence espagnole. La France et l'Espagne à l'époque d'Anne d'Autriche 1615-1666, Mont-de-Marsan, Éditions InterUniversitaires, 1991, pp. 39-51.

(10) Loubayssin, Engaños cit., Al discreto lector.

(11) «Sa langue est bonne, malgré quelques petits oublis»: A. Cioranescu, Le masque et le visage cit., p. 179.

(12) M. Pavesio, Primi embrioni pretirsiani del Don Giovanni, in M. Pavesio (dir.), Il convitato di pietra.

Don Giovanni e il sacro dalle origini al Romanticismo, Alessandria, Dell'Orso, 2002, pp. 7-25.

(13) Loubayssin, Engaños cit., p. 201.

(14) La precisazione si trova nella seconda parte del titolo. 
storico di Ercilla y Zuñiga sulla conquista del Cile ${ }^{15}$, e la componente esotica sarà in effetti valorizzata nel 1684 da un traduttore anonimo che denuncia un'infedeltà necessaria a «rendre parfait un Ouvrage qui ne l'étoit pas». Nella traduzione-rimaneggiamento Don Enrique de Castro, ou la conqueste des Indes, la galante schermaglia di armi e amori in sconfinati paesaggi australi presenta diverse avventure interpolate «tirées d'un autre Livre Espagnol, quoy que d'un stile corrompu, [qui] pourront divertir le Lecteur par leur variété»:

Enfin je n'ay rien oublié de ce que j'ay cru pouvoir rendre cette nouvelle agréable. Si les changements que j'y ay fait ne sont pas agréables à tout le monde, il y en aura d'autres qui auront plus d'indulgence, et m'auront obligation du dessein que $j$ 'ay eu de les divertir ${ }^{16}$.

D'altronde, in un contesto di scarsa propensione al riconoscimento autoriale, lo spirito di competizione mimetica che animava gli scrittori di corte non poteva che trovare fondamento e supporto in una delle figure retoriche ricorrenti nei testi liminari, la premunizione, in questo caso applicata da Loubayssin alla sua competenza linguistica:

Estimaré en mucho tus glosas, si como discreto amigo quieres enmendar las faltas, que por no saberlas conocer, avré dexado pasar, en esta primera impresion. [...] Que ya se sabe que no nacimos perfectos, sino para aprender todos los dias, por mucho que sepamos: Pues que Socrates con ser tan sabio, dixo estando al articulo de la muerte: que le pesava de morir, solo por ver que moria, quando empeçava a aprender ${ }^{17}$.

La ricezione francese di Engaños deste siglo è molto più complessa e articolata, a riprova del fatto che la consuetudine di «larcin à un bel esprit» denunciata da Bouhours verso fine secolo ${ }^{18}$ era già talmente diffusa da rendere arduo il tentativo di circoscriverne le modalità. La versione intitolata Les abus du monde, histoire mémorable de notre temps, pubblicata da François de Rosset nel 1618 e riproposta l'anno successivo da Du Bray, è nota anche con il titolo Histoire mémorable des tromperies de notre temps. La traduzione di de Rosset, «une histoire non moins remarquable pour ses accidens divers, que memorable pour les succez ordinaires qui procedent de pareils subiects», non menziona il nome dell'autore, appena evocato nella dedica che conferma il successo dell'originale spagnolo: «Un bel Esprit a pris la peine de l'escrire en Espagnol, et s'en est si bien aquité, qu'il n'y a rien à desirer: et sur son exemplaire je l'ai mise en nostre langue» ${ }^{19}$.

È sostanzialmente fedele all'originale, se non per alcune considerazioni volte a rendere «memorable» la vicenda narrata:

Et voila comme les accidents du monde sont divers: les tromperies estranges: les malheurs ordinaires, et la fortune muable. Ceste Histoire memorable autant peut-estre qu'autre qui soit arrivée en nos jours, le tesmoigne, si bien qu'admirant les abus de nostre siecle, et les changements qui succedent tous les jours aux affaires du monde. Je ne puis dire autre chose, sinon que

(15) Si tratta della Araucana di Alfonso de Ercilla y Zuñiga, pubblicato a Madrid tra il 1578 e il 1597. P. Neruda, Para nacer he nacido, Barcelona, Seix Barral, 1978, pp. 198-200.

(16) Anonimo, Dom Enrique de Castro ou la conqueste des Indes, Paris, de Luyne, 1684, Avis au lecteur (anche per le citazioni precedenti).

(17) Loubayssin, Historia tragicómica de Henrique de Castro, Paris, veuve de Mathieu Guillemot, 1617, Prólogo al lector.

(18) D. Bouhours, Les Entretiens d'Ariste et d'Eugène, Paris, Sebastien Mabre-Cramoisy, 1671, p. 200.

(19) F. de Rosset, Les abus du monde, Paris, de Rosset, 1618, dedica a M. De Chauvelin. 
tout ce qui est contenu sous l'Astre qui change de tant de formes nouvelles, ne demeure jamais en un mesme estat, et principallement les choses de l'Amour qui n'ont rien de veritable que les apparences, ny rien de plus constant que leur inconstance ${ }^{20}$.

Una traduzione di Deganes de Languedoc è poi pubblicata a Parigi da Hénault nel 1639, contemporaneamente all'uscita del panegirico, con il titolo Les tromperies de ce siècle. Nel Settecento il testo è ripreso con forti manipolazioni strutturali e interpolazione di commenti in una anonima Histoire des Cocus (Constantinople, 1741 e La Haye, 1746), presentata come «traduction française d'une nouvelle espagnole de Loubayssin de la Marca», e infine nel 1875 in Italia da un traduttore anonimo che però cita il nome dell'autore ${ }^{21}$.

In un certo senso, la fortuna di Engaños è paradigmatica di un momento storico e letterario in cui il mestiere di traduttore è assimilato a quello di grammatico in una dimensione pedagogica e divulgativa scarsamente riconosciuta ${ }^{22}$. I testi, di proprietà dei librai, potevano trasformarsi in «simples spéculations de librairie» ${ }^{23}$, la cui revisione, raramente eseguita dall'autore, convogliava distorsioni tanto impercettibili quanto sostanziali, fino allo stravolgimento proclamato dell'originale. La ricerca dell'equivalenza si articolava in una quantità di sfumature che dipendevano dalle richieste della committenza e del pubblico, dalla competenza del traduttore e dalla sua posizione sociale.

Il nome di Loubayssin non compare nei repertori dei traduttori professionisti e nemmeno tra gli scrittori di corte, tuttavia il successo di Engaños in Francia suggerisce la sua prossimità al milieu aristocratico e, probabilmente, una protezione altolocata: il commento sui matrimoni spagnoli che accompagna la dedica a Henri de Gondy, cugino del più famoso Cardinale di Retz, lo annovera tra i dignitari e cortigiani occupati a celebrare gli eventi reali per assicurarsi fama e reputazione; mentre la Historia tragicómica, «compuesta por Don Francisco Loubayssin de la Marca, Gentilhombre ordinario de la casa de dicho Señor» è dedicata a Don Luis de Lorena Cardinale di Guisa, ramo cadetto di una famiglia tradizionalmente vicina alla casa d'Austria.

Se i romanzi spagnoli sono riconducibili alla circolazione della novela del Siglo de Oro fuori dalla Spagna ${ }^{24}$, il terzo romanzo pubblicato incompiuto in francese nel

(20) Ivi, p. 153.

(21) L'edizione del 1746 è introdotta da una nota bio-bibliografica in cui si legge che «l'auteur, qui s'appelait Francisco Loubayssin de la Marca, publia dans la même ville d'autres romans d'aventures, mais celui-ci, à en juger par les traductions qu'il obtint, fut celui qui eut le plus de succès». Nella nota all'edizione italiana invece l'editore, ricordando le traduzioni precedenti, spiega che si tratta di una riduzione o imitazione anonima dell'originale: «traduction française d'une nouvelle espagnole de Loubayssin de la Marca». Per un'analisi delle traduzioni dell'opera di Loubayssin cf. S. Munari, L'opera bilingue di François Loubayssin de La Marque: un contributo all'ispanismo francese secentesco, in G. Bosco, M. Pavesio, L. Rescia (dir.), Contatti, passaggi, metamorfosi. Studi di letteratura francese e comparata in onore di Daniela Dalla Valle, Roma, Edizioni di storia e letteratura, 2010, pp. 51-67.

(22) Le versioni ad usum delphini, dalle raccolte di classici latini e greci destinate alla formazione dei prìncipi alle edizioni dei romanzi per l'insegnamento delle lingue straniere, alimentano l'idea che «traduire est chose vile», come afferma Chapelain nella dedica al lettore che apre Le Gueux, traduzione francese del Guzmán de Alfarache. Il capolavoro di Mateo Alemán gli era stato commissionato nell'ambito della sua attività di precettore, e Chapelain confessa di aver dovuto vincere la ripugnanza per il tipo di lavoro e il timore che la traduzione non potesse comunque avere il successo dell'originale. J. Chapelain, Le Gueux ou la vie de Guzman de Alfarache, Paris, Billaine, 1619, Au Lecteur.

(23) A. Cioranescu, Le masque et le visage cit., p. 179.

(24) La critica ha rilevato echi della Celestina, allegorie e digressioni moralizzanti che ricordano il Guzmán de Alfarache e l'Amadís de Gaula, riferimenti alle Crónicas e la presenza fra le righe di Lope de Vega. Cf. F. Castillo-Sánchez, La originalidad de "La bistoria tragicómica de don Henrique de Castro" (1617) de Francisco Loubayssin de la Marca, in Actas del XII Congreso de la Asociación Internacional de Hispanistas, Birmingham, 1998, https://cvc.cervantes.es/literatura/aih/pdf/12/aih_12_2_015. Dello stesso autore, 
1619 è invece ricordato negli studi secenteschi come «le Cid en France avant le Cid» ${ }^{25}$. Les Advantures béroyques et amoureuses du comte Raymond de Thoulouze et de Don Roderic de Vivar «que les Arabes appellerent Sid Campeador» ${ }^{26}$ mette in scena un celebre episodio della vita del Cid ed è uno dei primi esiti della fortuna della materia granadina in Francia ${ }^{27}$. Nel prologo Loubayssin suggerisce di avere una maggiore dimestichezza con la lingua spagnola senz'altro dovuta, oltre che alla lunga permanenza in Spagna, al fatto di parlare guascone, lingua del gruppo occitano-catalano molto diffusa a corte fin dall'epoca di Enrico IV ${ }^{28}$ :

Aussi ma main changeant de plume, ma bouche de langage, et mon esprit de dessein, s'est trouvé tellement confus lorsqu'il luy a fallu rompre les lois d'une si longue habitude (comme est celle que j'ay prise en composant mes livres Espagnols) que j'en ay perdu sinon tout à fait le jugement, à tout le moins l'esperance de te pouvoir donner ce Livre purement François ${ }^{29}$.

Il plurilinguismo di corte, legato alla presenza di Maria de' Medici e Anna d'Austria, contava anche la pratica delle lingue regionali, esito del delicato passaggio dal latino al francese. Con ogni evidenza Loubayssin scriveva in due lingue apprese in età adulta: «Me dezvanesco tanto en buscar umildades si escrivo en una lengua estrangera», afferma nella Historia tragicómica, mentre in Les advantures héroyques si avvertono i primi segni del nazionalismo culturale su cui poi prenderà corpo il panegirico dedicato a Richelieu: «Que si Mitridates aprit de parler Latin en faisant la guerre aux Romains avec l'espee, pourquoy ne croiras-tu pas que j'ay apris la langue Castillane en faisant la guerre aux Espagnols avec le cœur?» ${ }^{30}$.

Nel romanzo francese l'argomentazione dell'Advertissement ricalca il topos di una superiorità culturale nei confronti di un paese il cui ruolo politico internazionale suscita timore, quindi Loubayssin considera il francese più adatto a sostenere le regole della conversazione mondana non solo per le sue caratteristiche intrinseche, ma perché gli spagnoli hanno la pessima abitudine di ricordare all'interlocutore le loro conquiste militari. Tuttavia - finzione nella finzione - subordina la questione linguistica al discorso traduttivo, fingendo che Les advantures héroyques sia la traduzione di un manoscritto arabo:

Ie le devois plustot tourner en espagnol qu'en françois; car outre que la phrase arabe s'appoche plus de la castellane, que de la nostre, ie m'assure que ie l'eusse habillé si somptueusement à l'espagnolle, qu'on n'eut pas eu moins de plaisir d'en admirer les perfections, qu'on aura de mécontentement d'en connoistre les defauts. Ce n'est pas que ie veuille dire, que la langue française n'eut pu relever aussi bien le lustre de ceste traduction, que la castillane: car n'estimant rien tant après le bien que Dieu m'a fait me faisant naistre Chrestien, que d'estre né

cf. Influencias celestinescas en "La historia tragicómica de don Henrique de Castro" (1617) de Francisco Loubayssin de la Marca, https://cvc.cervantes.esliteratura/aih/pdf/13/aih_13_1_057. Si veda inoltre A. Pacheco-Ransanz, Francisco Loubayssin de Lamarca: notas para la bistoria de la novela española del Siglo de Oro, in Actas del Sexto Congreso de la Asociación Internacional de Hispanistas, Toronto 22-26 agosto 1977, Toronto, University of Toronto, 1980, pp. 553-557.

(25) G. Reynier, Le Cid en France avant le Cid, in Mélanges offerts par ses amis et élèves à G. Lanson, Genève, Slatkine, 1972.

(26) Loubayssin, Les Advantures héroyques et amoureuses du comte Raymond de Thoulouze et de Don Roderic de Vivar, Paris, du Bray, 1619, Advertissement.

(27) S. Munari, Il mito di Granada nel Seicento. La ricezione italiana e francese, Alessandria, Dell'Orso, 2002, pp. 111-114.

(28) A. Rey, F. Duval, G. Siouffi, Mille ans de langue française, histoire d'une passion, t. I, Paris, Perrin, 2011, p. 484.

(29) Loubayssin, Les Advantures cit., Advertissement.

(30) Ivi, Advertissement. 
français, i'ai tellement affectionné ce qui me fait estre ce que ie suis, que si ie n'ai pu acquerir la perfection d'ecrire bien en ma langue, ie l'ai acquise de savoir qu'estant aussi riche que la grecque, et aussi majestueuse que la latine, elle est sans comparaison plus belle et plus douce que toutes les autres ${ }^{31}$.

Nella precaria dimensione di soggezione cortigiana e clientelare in cui Loubayssin vive, la scelta linguistica sembra trasformarsi in luogo di identificazione e rivendicazione identitaria («ce qui me fait estre ce que ie suis»). Ė molto probabile che desiderasse affrancarsi dallo scomodo legame con i Guisa, attivi sostenitori di Maria de' Medici durante la reggenza e fin dal 1616 coinvolti nelle cospirazioni ai danni di Luigi XIII: strumento dell'ingerenza spagnola in Francia, reggevano le fila del malcontento della nobiltà che si riconosceva nel fratello del re Gaston d'Orléans ${ }^{32}$. Nel fluido rimescolarsi di alleanze, Loubayssin ritiene allora opportuno passare al francese per sottolineare la sua origine: «Les gascons n'ont jamais esté bons amis des Espagnols pour te donner sujet d'avoir ce soupçon de moy» ${ }^{33}$.

Tra la nobiltà filospagnola circolavano molti pamphlet antifrancesi, come il famoso trattato di Carlos García del 1617, La oposición y conjunción de los dos grandes Luminares de la Tierra o La Antipatía de Franceses y Españoles, che collocava la rivalità tra i due Paesi nell'ordine naturale dell'universo ${ }^{34}$. Il tema del carattere delle nazioni, poi ampliato alla dimensione linguistica in termini di "genio" della lingua, diventa in seguito uno dei fondamenti della propaganda antispagnola in Francia, soprattutto a partire dal 1635 con la nuova guerra. Nel 1639 il panegirico francese vi fa esplicita allusione:

Si disputer encore de la gloire, apres avoir merité l'honneur du Triomphe est mettre à la direction de la Fortune ce qui a esté des-ja mis à la direction d'un bon sens, je pense, mon cher Lecteur, que vous trouverez estrange, que soubs l'effort d'une vertu si faible que la mienne, j'aye pris la hardiesse de mettre sur les rangs, les deux plus belles Langues de l'Univers, pour faire connoistre à toute l'Europe, les avantages que l'une peut avoir sur l'autre, à escrire les Eloges, et les Panegiriques qui se font à la memoire des grands Roys, et des grands Ministres d'Estat"

L'unione matrimoniale del 1615 era stata per Richelieu e Olivares un'insperata occasione di carriera, ma le due Corone, entrambe decise a legittimarsi come garanti della pace europea, scontavano una frammentazione interna che impediva ai rispettivi ministri di incanalare le spinte antagoniste a sostegno dei loro obiettivi. Lo zelo riformista del Conte-duca sfidava l'inerzia di un'aristocrazia contraria a qualsiasi forma di rinnovamento, e subiva una cronica mancanza di denaro che limitava le ambizioni di conquista. Quanto a Richelieu, dopo la fuga di Gaston d'Orléans in Lorena nel 1631 si trovava in una situazione particolarmente complessa, alle prese con un paese disgregato dalle tensioni religiose e messo in pericolo dalla faziosità dell'alta aristocra$\mathrm{zia}^{36}$, come Loubayssin rileva sul filo della metafora:

(31) Ivi, Advertissement.

(32) A. Hugon, Au service du roi catholique. «Honorables ambassadeurs» et «divins espions», Madrid, Casa de Velázquez, 2004, p. 329.

(33) Loubayssin, Les Advantures cit., Advertissement.

(34) C. García, La oposición y conjunción de los dos grandes Luminares de la Tierra, o La Antipatía de Franceses y Españoles, éd. M. Bareau, Edmonton, Alta Press, 1979.

(35) Loubayssin, Deffy cit., Advertissement.

(36) J.H. Elliott, Richelieu e Olivares, Torino, Einaudi, 1990, p. 150. 
Je n'ay point d'exercice meilleur que la contemplation des Vertus admirables de Vostre Eminence. Ce sont elles, Monseigneur, qui par un nombre infiny de belles et grandes Actions, faisant refleurir en France la Sagesse, et la Valeur, que la negligence du Temps, et la confusion de l'Estat y avoient laissé flétrir depuis tant d'années la rendent aujourd'huy triomphante, et paisible Maistresse des Peuples et des Nations, sous le Regne, du plus Grand, du plus Iuste, et du plus Victorieux Prince qui n'ait jamais porté Couronne ${ }^{37}$.

Nel quadro di una politica estera che aveva come assoluta priorità il contenimento delle ambizioni francesi, Orléans, aspirante erede in esilio, non ottenne dagli spagnoli che un tiepido sostegno ${ }^{38}$. Poco dopo, all'apertura formale delle ostilità, i due ministri si circondarono di una vera e propria squadra di pubblicisti. Le tappe del conflitto sono contrassegnate da una pamphlettistica che alterna la denuncia del nemico alla propaganda nazionalista: i polemisti spagnoli contestano la tesi secondo cui la Spagna aspirerebbe alla monarchia universale, e giustificano la necessità di una guerra difensiva, mentre i francesi coltivano un'immagine di perseveranza e lungimirante disinteresse in nome di una superiore ragione di Stato.

Il Deffy partecipa alla creazione di una «fiction publique du discours» ${ }^{39}$ le cui coordinate affondano nel gioco retorico della personificazione che mescola tratti linguistici e peculiarità morali: se lo spagnolo appare esagerato e rigido, l'italiano è lezioso e desideroso di piacere, mentre il francese è bello e dolce, ricco come il greco e grave come il latino. Sono luoghi comuni già presenti nell'opera di Henri Estienne e nei trattati teorici sull'antipatia tra le nazioni, cliché culturali che dopo il 1635 risuonano nella letteratura antispagnola dissimulata in questioni di stile ${ }^{40}$ :

Il ne seroit point juste que la Langue Françoise, qui est aussi riche que la Greque, et aussi grave que la Latine, et par consequent plus belle et plus douce que toutes les autres, se mit en lice pour disputer de la gloire de l'Eloquence, avec l'Espagnole, qui luy est de beaucoup inferieure, quoy qu'on die, en grace, en force, et en elegance ${ }^{41}$.

Nella rielaborazione encomiastica il confronto si concentra sui due protagonisti: Filippo IV, paladino del'ortodossia cristiana, combatte non per estendere i propri domini ma per conservare l'ordine europeo che la Francia è determinata a sovvertire; Luigi XIII, attraverso Richelieu, predica il primato della ragione mitigata da quella prudenza che gli ha permesso di liberarsi degli oppositori interni (Gaston d'Orléans, i Guisa e la regina madre) confinati in Lorena e nei Paesi Bassi spagnoli ${ }^{42}$ :

L'esprit travaille, et le Corps agit; Pour estre un grand Chef, il faut estre un fort Homme; et l'esprit ne peut pas bien conduire un Estat, si l'on n'a la main assez forte pour executer: Et encor que les momens de vostre repos soient autant de pertes que fait la France; Il est bon quelque fois de s'arrester, pour mieux courir; d'autant que fuyr les conseils precipitez, c'est discourir pour atteindre aux Conseils bien pris. Vostre personne nous estant si chere, Monseigneur, il n'est point juste que vous vous pressiez, pour des conquestes qui ne nous peuvent fuyr qu'avec vostre vie. Le Roy fera bien, tandis que vous ne ferez point mal; Car la pesanteur de sa

(37) Loubayssin, Deffy cit., pp. 1-2.

(38) J.H. Elliott, Richelieu e Olivares cit., p. 111.

(39) G. Siouffi, L'apologétique cit., p. 176.

(40) L. Van Delft, Espaces, frontières et anthropologie: les caractères des nations à l'âge classique, in R. Bauer, D. Wessel Fokkema, M. de Graat (dir.), Space and boundaries - Espace et Frontières, t. II, München, Iudicium Verlag, 1990, pp. 130-136.

(41) Loubayssin, Deffy cit., Advertissement.

(42) J.H. Elliott, Il miraggio dell'impero. Olivares e la Spagna: dall'apogeo al declino, t. II, Roma, Salerno, 1991, p. 21 e p. 585. 
charge est bien grande, pour croire qu'autre que vous le puisse secourir de son ayde. Il confie à vostre soin, ce que vostre soin confie à la Sagesse, et à la Prudence, que toutes les puissances de la terre ne sçauroient trouver chez eux, au point que sa Majesté les trouve chez vous ${ }^{43}$.

A Madrid Olivares recluta Quevedo, autore del famoso Visite et anatomie de la tête du Cardinal de Richelieu, e sceglie Virgilio Malvezzi come storico ufficiale del regno. Il progetto di una nuova Storia spagnola destinata alla pubblicazione in quattro lingue (spagnolo, francese, latino e italiano) non è portato a termine, ma si moltiplicano i manifesti bilingui ${ }^{44}$ che individuano nel carattere nazionale l'elemento chiave del successo politico e militare ${ }^{45}$.

Sensibile alle potenzialità del linguaggio come strumento di controllo, anche Richelieu corteggia gli intellettuali: può contare sull'appoggio della Gazette di Renaudot e fonda l'Académie per trasformare il francese in lingua elitaria secondo l'ideale retorico dell'umanesimo, legittimandola come lingua "viva" destinata alla rappresentazione del potere taumaturgico del re. Promuove una politica linguistica in cui l'idea di perfezione della lingua reale, concepita in funzione celebrativa, si esprime nella ricerca di una nuova eloquenza che coniughi «simplicité, facilité, clarté» a «majesté» e «sublime» ${ }^{46}$.

Nella Babele di corte, dove coesistono latino, parlate regionali e le lingue portate in Francia dalle regine straniere, un mondo chiuso alla ricerca di nuovi modelli di riferimento ${ }^{47}$, prende corpo una riflessione sulle regole del bel usage e sulla traduzione, si pubblicano trattati, dizionari e grammatiche, gli autori sono chiamati a giustificare le loro scelte linguistiche. In un quadro di tale ambiguità e incertezza il panegirico di Loubayssin si allinea alla tradizione classica che aveva ispirato autori illustri: due anni prima Mascaron aveva dedicato al ministro La mort et les dernières paroles de Sénèque, mentre La Mothe Le Vayer aveva pubblicato nel 1636 il Discours de la contrariété d'bumeurs qui se trouve entre certaines nations et singulièrement entre la Françoise et l'Espagnole (che finge di tradurre dall'italiano ma è un plagio dell'opera di García) e nel 1638 Considérations sur l'éloquence françoise de ce temps ${ }^{48}$.

Nonostante i successi in battaglia, nel 1639 l'esercito è allo stremo, la Spagna sta attraversando una fase drammatica, Olivares può contare solo su un indebolimento francese generato dalle divisioni interne. In Francia, dove Mazarino sta per sostituire Richelieu malato, il momento è quanto mai delicato:

Continuez, Monseigneur, à nous rendre heureux par les Victoires que vous gaignez quand il vous plaist, et que vous donnés à qui bon vous semble. Nous ne craignons point que vous changiez d'affection; mais nous craignons que vous manquiez de santé. Toutesfois vostre corps

(43) Loubayssin, Deffy cit., pp. 6-7.

(44) Nel 1635 Céspedes pubblica Francia engañada, Francia respondida - France trompée et France instruite mentre Pellicer de Tovar scrive nel 1639 El verdadero católico de Estado y lágrimas de EuropaL'anticatholique d'Etat et les larmes d'Europe. L'anno precedente il pamphlet di Carlos García era stato ristampato con traduzione francese a fronte, e nel 1639 Malvezzi, ormai entrato nel Consiglio di guerra del re, scrive La libra traducida de italiano en lengua castellana che stamperà con lo pseudonimo di Grivilio Vezzalmi a Pamplona nel 1640. Anche Successi principali della monarchia di Spagna nell'anno 1639 esce in spagnolo nel 1640, anno in cui Malvezzi fu nominato ambasciatore in Inghilterra, e poi in italiano ad Anversa nel 1641. Cf. A. Hugon, Philippe IV, Paris, Payot, 2014, p. 192.

(45) J.H. Elliott, Il miraggio dell'impero cit., p. 120.

(46) M. Fumaroli, L'apologétique de la langue française classique, "Rhetorica" 1984/II, p. 156.

(47) A. Rey, F. Duval, G. Siouff, Mille ans de langue française cit., p. 417.

(48) Cf. J.L. Colomer, La France et l'Espagne en guerre: Virgilio Malvezzi dans la polémique française sur le style «coupé», in C. Mazouer (dir.), L'âge d'or de l'influence espagnole cit., pp. 229-240; A. Mansau, L'espagnolisme, cette façon de sentir, ivi, pp. 209-217; S. Murr, Piété espagnole, vertu des indiens et piété française: une métaphore du libertinisme chrétien chez La Mothe Le Vayer, ivi, pp. 426-439. 
né à faire toute sorte d'exercices de Paix et de Guerre, ayant esté attacqué par les maladies, en a surmonté glorieusement les hazards, par le bonheur de la complexion forte et vigoureuse, et le secours de son courage grand et admirable. Il a esté poussé, non pas abattu, puis que se tenant tousiours ferme, il s'est maintenu droit sur ses pieds, faisant connoistre à l'assaillant par sa resistence, qu'il ne seroit jamais vaincu par la force; et qu'il n'apartenoit point à une fièvre causee par un peu de sang corrompu, de ranger sous ses Loix celuy que la Nature a fait naistre, pour gouverner tout le Monde ${ }^{49}$.

Loubayssin mette il suo bilinguismo di scrittura al servizio della causa francese per esprimere «l'extreme affection que j'ay pour ma Patrie» e contribuire «à la Gloire de mon Pays, par cette adresse, dont je me sers pour relever le lustre, et la Maiesté de sa Langue $»^{50}$. Elogia il francese secondo una prassi collaudata in cui l'eleganza formale fa risaltare la consapevolezza delle potenzialità espressive:

Et ay si hautement poursuivy le cours de mon dessein, rehaussant en François le lustre que la force du Castillan n'a peu perfectioner en sa langue, qu'il est bien difficile d'en voir les traits, qu'on ne dise aussi-tost, que celuy-là connoist bien le merite du sujet, qui le sçait traiter avec tant d'Eloquence, et que pour devenir grand Maistre à bien faire, il n'est rien de meilleur, que de travailler incessamment pour ne point faillir ${ }^{51}$.

Esaltare la lingua per onorare il re: poiché l'apologetica della corona si specchia nella gloria della lingua reale, l'obiettivo dichiarato è dimostrare come il francese sia di gran lunga più adatto dello spagnolo al genere celebrativo. Per vincere il nemico - diceva César Oudin nella prefazione alla Grammaire espagnolle (1596) - bisogna conoscerlo e colpirlo con i suoi mezzi, un'idea condivisa da Loubayssin nell'Advertissement au Lecteur che introduce il Deffy:

Et quoy qu'il soit difficile que l'insuffisence de l'Ouvrier ne rende souvent une belle matiere sterile à produire de grands effects; je vous donneray pour raison d'avoir heureusement vaincu ceste difficulté, l'extreme affection que j'ay pour ma Patrie, et le secret particulier que l'Espagne a fait avoir à ma plume (comme vous avez peu voir par les livres Castillans que j'ay donnez au public) de rabaisser aussi justement nos ennemis par leur propre langue, que nous les avons battus courageusement par nos seules Armes ${ }^{52}$.

Nel Prologo de la Lengua Española, al discreto Lector che accompagna il Repto, invece, Loubayssin afferma che uno scrupolo di coscienza lo costringe a paragonare «la equidad de las armas de Francia contra España», laddove per "armi” si intendono la coerenza retorica, la padronanza stilistica e l'agilità oratoria. La sua voce si sdoppia in una ricerca di eloquenza che adegua il modello ciceroniano di totale fiducia nel prestigio e nel potere della parola a una nuova cultura di corte, «où l'art de réussir parmi les passions et les vicissitudes de la fortune - ricorda Fumaroli - est d'abord un art de bien dire ce qu'il faut au bon moment et au bon endroit» $\gg^{53}$ :

Siguo de manera el intento del que me pone en obra, que si no salgo por mis cabales en la Eloquencia que pretendo, doy tanto que correr a la Lengua Francesa, que no se si me alcançara

(49) Loubayssin, Deffy cit., pp. 4-5.

(50) Ivi, Advertissement.

(51) Ibidem.

(52) Ibidem.

(53) M. Fumaroli, L'âge de l'éloquence, Rhétorique et «res literaria» de la Renaissance au seuil de l'époque classique, Paris, Albin Michel, 1994, p. 45. 
mientras uviere Escritores. [...] Vaya el Panegirico, que una continua admiracion de perfecta Virtud, me hizo escrevir con pluma de Frances, y estilo de Castellano atrevido y raro: que despues de cotejado y bien medido con los Concetos, Frasis, Razones, Fuerças y Gracias del Lenguaje del de mi Parte, a guardar con mucha constancia la Sentencia; o para seguir el Carro de Triumpho, como Vencido, o para Triumphar en el, como Vencedor ${ }^{54}$.

In entrambe le versioni, il panegirico applica i principi della techne epidittica: nascondere i difetti o trasfigurarli in qualità, anche inventate, e ricorrere alla deformazione o rilettura dei fatti storici sottolineando la propria sincerità e l'insufficienza dell'esercizio retorico a fronte della grandezza dell'azione politica:

Estant plus véritable qu'éloquent, je ne me sçaurois étudier à prouver par des parolles, ce que mon Prince fait voir par ses actions. [...] Ce n'est pas neantmoins avec ce discours, Monseigneur, mais avec celuy d'un autre qui seroit aussi éloquent que ie suis affectionné, qu'on pourroit dire la verité de tres-bonne grace; que l'honneur des hommes se pourroit rendre pareil à celuy des Dieux, et que l'éclat des choses presantes, ne sçauroit estre offusqué par la beauté des choses futures 55 .

La trasformazione della Storia in storie ad opera dell'immaginazione, la fiction che dà forma al potere, passa per una serie di metafore sulle quali lo stesso Richelieu ha costruito la propria immagine ${ }^{56}$ : mentre la figura del re è evocata mediante rimandi mitologici e allegorie astrali, il campo semantico riservato al suo ministro ruota principalmente intorno agli elementi del corpo (nelle fasi di forza, crescita e salute ma anche di malattia, declino, abbandono), e del viaggio nella tormenta in cui il nocchiero riesce a condurre lo Stato a un porto sicuro:

Souvenez-vous, Monseigneur, qu'il n'appartient qu'à l'Eminence du grand Cardinal Duc de Richelieu, d'élever des Temples sur leurs ruynes à l'honneur de son Prince; Et que si tant de grands hommes qui se sont perdus avec leur pays, preferant le bien public à leur interest particulier, eussent fait autant d'estat de la conservation et du soulagement de leur Patrie, que de la haine et de l'envie de leurs ennemis; tenant ferme au maniment des affaires, comme fait le Pilote au gouvernail en temps de tourmante; ils n'eussent pas seulement dissipé l'orage qui les menaçoit, et conduit l'Estat à bon port, mais acquis comme vous, Monseigneur, Vostre Eminence, loin du naturel farouche de cet Athenien, qui s'estoit declaré ennemy de tous les hommes, ne veut pas seulement estre bien avec ceux qui l'ayment, mais encor avec ceux qui le hayssent $t^{57}$.

Ma nelle due lingue l'esercizio politico assume sfumature differenti: in francese si elencano i successi militari contro la casa d'Austria e si sottolinea la capacità del sovrano di delegare e confrontarsi; in spagnolo si illustra la politica estera del Cardinale che ha firmato alleanze con Inghilterra e Svezia per rompere l'assedio asburgico e si ricorda la recente nascita del delfino Luigi XIV, promessa di continuità e sicurezza:

Si el sucesso no corresponde a mi opinion, escusareme Señor, con dezir que quen se emplea en cosas tan altas, no deve ser falto de atrevimiento ni de Animo; y que quando esta desdicha me viniere por no tener harta siencia, que la passion que tengo de morir a los pies de

(54) Loubayssin, Repto entre la lengua española y la francesa, Paris, Morlot, 1639, Prologo de la Lengua Española, al discreto Lector.

(55) Loubayssin, Deffy cit., p. 2 e p. 15.

(56) J.-P. Étienvre, Pour une histoire de la métaphore politique, in J.-P. Étienvre (dir.), Littérature et politique en Espagne aux siècles d'or, Paris, Klincksieck, 1998, p. 13 e pp. 16-18.

(57) Loubayssin, Deffy cit., p. 8. 
Vuessa Eminencia, viendola venir vitoriosa de una batalla que de a mi Rey el Imperio de toda la Tierra, pues la Reyna nos ha dado estos dias un Delphin que le podra dichosamente succeder, estando en su perficion mas complida, bastara para todo. Guardeme Dios a Vossa Eminencia, y prospere largos años en su Santo Servicio ${ }^{58}$.

Se il Deffy è del tutto encomiastico, nella riscrittura l'autore ripercorre le ambizioni imperiali della Spagna, esprime un giudizio favorevole sui primi dieci anni di governo di Richelieu e chiarisce i motivi che hanno costretto la Francia a entrare in guerra. Il Cardinale, cui si deve una Francia pacificata e arbitra d'Europa, è opposto all'Olivares che ha portato al disfacimento una nazione potente: la loro rivalità personale, nutrita dello stesso marchio di arroganza che molto contribuiva alla loro impopolarită ${ }^{59}$, diventa la chiave di rappresentazione del conflitto tra i due Paesi, messo in scena con un espediente tipico del genere moresco. In luogo della battaglia si sfidavano in torneo un comandante moro e un cavaliere cristiano, così che l'eroe positivo potesse emergere grazie al co-protagonista:

Don Roderic qui n'eust iamais creu, que son ennemy eust esté roy, et celui pour qui il avoit entrepris son voyage, demeura le plus estonné du monde, et encore plus de voir en luy, une si grande courtoisie, veu la cruauté avec laquelle il l'avoit traicté: il s'approche donc de luy $[\ldots]$ s'excuse sur son ignorance, luy demande pardon, et luy donne avec toutes les courtoisies du monde, la gloire du combat ${ }^{60}$.

Nel romanzo sul Cid Raymond de Thoulouze e Roderic de Vivar si affrontano secondo le regole; nei due panegirici si scontrano Richelieu, artefice del ritrovato splendore della corona francese, e don Gaspar de Guzmán, una delle personalità politiche più interessanti e controverse del Seicento europeo. Loubayssin sembra applicare i principi dell'exemplum, dell'etopea e della demonstratio propri della comunicazione oratoria - e anche della drammaturgia secentesca, ricorda Fumaroli - che componeva "maschere" di personalità celebri o "caratteri" morali adattati alle esigenze della verosimiglianza ricorrendo a un «universale dell'immaginario» condiviso dal pubblico ${ }^{61}$.

La figura di Loubayssin, un autore di fatto trilingue alle prese con gli equilibri sfuggenti della politica e le dinamiche ambigue della cortigianìa, risulta assai moderna nella ricerca di un'appartenenza linguistica. Con una prudente strategia di ridefinizione del rapporto mecenatesco, nella fase più acuta del conflitto tra i due Paesi ricorre a un esercizio di retorica e adulazione per riformulare il récit du roi tramite la pratica traduttiva, strumento duttile e flessibile. Rivendica la conoscenza dello spagnolo, lingua in cui aveva avuto successo come autore, per rimarcare l'aderenza alla cultura francese, e l'atto traduttivo sembra inteso come uno spazio utile a fissare le coordinate di un'identità confusa (nei repertori bibliografici il suo nome compare alternativamente nelle due lingue). Quando spiega di aver tradotto il panegirico in castigliano affinché gli spagnoli leggano nella loro lingua «iusques dans le Mexique partie des actions de ce grand Genie, qui ayant ébranlé leur superbe Empire, ils nomment Demonio, écrites par la main d'un François» ${ }^{62}$, dobbiamo immaginare che essere «sujet du roi de France» - così si definiva - gli garantisse un ruolo nel mondo

(58) Loubayssin, Repto cit., p. 21.

(59) J.H. Elliott, Richelieu e Olivares cit., p. 48.

(60) Loubayssin, Les Advantures cit., p. 48.

(61) M. Fumaroli, Eroi e oratori. Retorica e drammaturgia secentesche, Bologna, il Mulino, 1990, p. 46.

(62) Loubayssin, Deffy cit., Advertissement. 
chiuso e protetto dell'encomiastica reale, in un chiasmo evidente tra potere politico e potere del discorso:

Pues que un entendimiento arto comun como el mio, se adelgaza de manera, en solo considerar quan adorables son la Maravillas que en menos de 10 años su dichoso Govierno ha derramado sobre el Teatro universal de toda la Europa, que con humo estraño, echando con ostentacion de ingenio un Repto entre la lengua Espanola, y Francesa, para ver cual de las dos sera mas Eloquente, en dezirlas, pretende ganar una Gloria Inmortal alcanzando de Vuessa Eminencia la aprobacion de todas las $\operatorname{dos}^{63}$.

Sorge allora spontaneo chiedersi, anche di fronte a una figura marginale come quella di Loubayssin, se la dimensione di conquista, il carattere «predatorio» ${ }^{64}$ che accompagna la creazione letteraria secentesca e la creazione di una teoria della lingua volta a promuovere l'egemonia politica e culturale sia da intendersi sul piano storicoletterario solo come momento di costruzione di una dimensione culturale che sfocerà nel rayonnement europeo settecentesco, o non dia invece vita a un movimento vertiginoso di sovrapposizione tra lettura e scrittura che in qualche modo, ancora oggi, si mantiene nella predisposizione della lingua francese a riflettere su se stessa attraverso i suoi autori. 